\title{
Ricci Solitons on Lorentzian Four-Dimensional Generalized Symmetric Spaces
}

\author{
Amel Bouharis and Bachir Djebbar
}

The existence of non-trivial, i.e., non-Einstein, Ricci solitons on fourdimensional Lorentzian generalized symmetric spaces is proved. Moreover, it is shown that only steady Ricci solitons can be gradient.

Key words: Lorentzian metric, Ricci solitons, gradient Ricci solitons, generalized symmetric spaces.

Mathematical Subject Classification 2010: 53C20, 53C21.

\section{Introduction}

A Ricci soliton is a natural generalization of an Einstein metric. It is defined on a pseudo-Riemannian manifold $(M, g)$ by

$$
L_{X} g+\varrho=\lambda g,
$$

where $X$ is a smooth vector field on $M, L_{X}$ denotes the Lie derivative in the direction of $X, \varrho$ is the Ricci tensor and $\lambda$ is a real number. Moreover, we say that the Ricci soliton $(M, g)$ is a gradient Ricci soliton if it admits a vector field $X$ satisfying $X=\operatorname{grad} h$ for some potential function $h$.

A pseudo-Riemannian manifold $(M, g)$ is said to be Yamabe soliton if it admits a vector field $X$ such that

$$
L_{X} g=(\tau-\gamma) g,
$$

where $\tau$ denotes the scalar curvature of $(M, g)$ and $\gamma$ is a real number.

A Ricci soliton (Yamabe soliton) is said to be shrinking, steady or expanding according to whether $\lambda>0, \lambda=0$ or $\lambda<0$ if $\gamma>0, \gamma=0$ or $\gamma<0$, respectively.

In [4], the authors studied Ricci and Yamabe solitons on second-order symmetric Lorentzian spaces.

Lorentzian Ricci solitons have been intensively studied showing many essential differences with respect to the Riemannian case [2,8,14]. In fact, although there exist three-dimensional Riemannian homogeneous Ricci solitons $[1,13]$, there are no left-invariant Riemannian Ricci solitons on three-dimensional Lie groups [11]

(C) Amel Bouharis and Bachir Djebbar, 2018 
(see also [15]). Moreover, the Lorentzian case is much richer, allowing the existence of expanding, steady and shrinking left-invariant Ricci solitons [5]. Recently, Calvaruso and Fino proved, contrarily to the Riemannian case, the existence of non-compact four-dimensional homogeneous pseudo-Riemannian Ricci solitons which are not isometric to solvmanifolds [9]. These results make it interesting for further investigation of Ricci solitons on Lorentzian manifolds.

In [12], pseudo-Riemannian four-dimensional generalized symmetric spaces were classified into four classes, named A, B, C and D, and the pseudoRiemannian metrics can have any signature. All these spaces are reductive homogeneous. Their geometrical properties were studied by Calvaruso and De Leo in [7].

Four-dimensional generalized symmetric spaces were studied by many authors. In particular, in [3], the authors classified, up to isometry, non-symmetric simply-connected four-dimensional pseudo-Riemannian generalized symmetric spaces which are algebraic Ricci solitons. Those of Cerny-Kowalski's types A, C and D are algebraic Ricci solitons, whereas those of type B are not.

A complete classification of Ricci solitons of generalized symmetric spaces of type A, B, D has been recently obtained in [10], leading to new examples. In this paper, we find the general solution to equation (1.1) for generalized symmetric spaces of type C.

The paper is organized in the following way. In Section 2, we shall report the basic description of four-dimensional generalized symmetric spaces. In Section 3, the Levi-Civita connection, the curvature tensor and the Ricci tensor of Lorentzian four-dimensional generalized symmetric spaces will be described in terms of components with respect to the coordinate vector fields $\left\{\partial_{i}=\frac{\partial}{\partial x_{i}},\right\}$. This provides the needed information for the study, which we do in Section 4. In Section 4, Ricci solitons on generalized symmetric spaces of type $\mathrm{C}$ are characterized via a system of partial differential equations. In particular, we show that these spaces admit different vector fields resulting in expanding, steady and shrinking Ricci solitons. Finally, it is proved that those Ricci solitons are gradient only in the steady case.

\section{Four-dimensional generalized symmetric spaces}

We start by recalling the definition of the generalized symmetric space. Let $(M, g)$ be a (pseudo-)Riemannian manifold. A regular $s$-structure on $M$ is a family of isometries $\left\{s_{p} \mid p \in M\right\}$ of $(M, g)$ such that

- the mapping $M \times M \rightarrow M,(p, q) \mapsto s_{p}(q)$, is smooth,

- $p$ is an isolated fixed point of $s_{p}, \forall p \in M$,

- $s_{p} \circ s_{q}=s_{s_{p}(q)} \circ s_{p}, \forall p, q \in M$.

The map $s_{p}$ is called the symmetry centered at $p$. The order of a regular $s$ structure is the smallest integer $k \geqslant 2$ such that $s_{p}^{k}=\mathrm{id}_{M}$ for all $p \in M$. If such an integer does not exist, we say that the regular $s$-structure has order infinity. A 
generalized symmetric space is a connected pseudo-Riemannian manifold carrying at least one regular $s$-structure. In particular, a generalized symmetric space is a pseudo-Riemannian symmetric space if and only if it admits a regular $s$-structure of order 2. The order of a generalized symmetric space is the minimum of orders of all possible $s$-structures on it. Furthermore, if $(M, g)$ is a generalized symmetric space, then it is homogeneous, that is, the full isometry group $I(M)$ of $M$ acts transitively on it, which means that $(M, g)$ can be identified with $(G / H, g)$, where $G \subset I(M)$ is a subgroup of $I(M)$ acting transitively on $M$, and $H$ is the isotropy group at a fixed point $o \in M$.

Generalized symmetric spaces of low dimension have been completely classified. The following theorem recalls the classification of non-symmetric simplyconnected 4-dimensional pseudo-Riemannian generalized symmetric spaces.

Theorem 2.1 (Cerny and Kowalski [12]). Non-symmetric, simply-connected generalized symmetric spaces $(M, g)$ of dimension 4 are of order either 3 or 4 , or infinity. All these spaces are indecomposable and belong, up to isometry, to one of the following four types.

Type A. The underlying homogeneous space is $G / H$, where

$$
G=\left(\begin{array}{ccc}
a & b & x_{3} \\
c & d & x_{4} \\
0 & 0 & 1
\end{array}\right), \quad H=\left(\begin{array}{ccc}
\cos t & -\sin t & 0 \\
\sin t & \cos t & 0 \\
0 & 0 & 1
\end{array}\right)
$$

with $a d-b c=1 . \quad(M, g)$ is the space $\mathbb{R}^{4}\left(x_{1}, x_{2}, x_{3}, x_{4}\right)$ with the pseudoRiemannian metric

$$
\begin{aligned}
g= & \lambda\left[\left(1+x_{2}^{2}\right) d x_{1}^{2}+\left(1+x_{1}^{2}\right) d x_{2}^{2}-2 x_{1} x_{2} d x_{1} d x_{2}\right] /\left(1+x_{1}^{2}+x_{2}^{2}\right) \\
& \pm\left[\left(-x_{1}+\sqrt{1+x_{1}^{2}+x_{2}^{2}}\right) d x_{3}^{2}\right. \\
& \left.+\left(x_{1}+\sqrt{1+x_{1}^{2}+x_{2}^{2}}\right) d x_{4}^{2}-2 x_{2}^{2} d x_{3} d x_{4}\right],
\end{aligned}
$$

where $\lambda \neq 0$ is a real constant. The order is $k=3$, and the possible signatures are $(4,0),(2,2)$ and $(0,4)$.

Type B. The underlying homogeneous space is $G / H$, where

$$
G=\left(\begin{array}{cccc}
e^{-\left(x_{1}+x_{2}\right)} & 0 & 0 & a \\
0 & e^{x_{1}} & 0 & b \\
0 & 0 & e^{x_{2}} & c \\
0 & 0 & 0 & 1
\end{array}\right), \quad H=\left(\begin{array}{cccc}
1 & 0 & 0 & -w \\
0 & 1 & 0 & -2 w \\
0 & 0 & 1 & 2 w \\
0 & 0 & 0 & 1
\end{array}\right) .
$$

$(M, g)$ is the space $\mathbb{R}^{4}\left(x_{1}, x_{2}, x_{3}, x_{4}\right)$ with the pseudo-Riemannian metric

$$
\begin{aligned}
g=\lambda\left(d x_{1}^{2}+d x_{2}^{2}+d x_{1} d x_{2}\right) & +e^{-x_{2}}\left(2 d x_{1}+d x_{2}\right) d x_{4} \\
& +e^{-x_{1}}\left(d x_{1}+2 d x_{2}\right) d x_{3},
\end{aligned}
$$

where $\lambda$ is a real constant. The order is $k=3$, and the signature is always $(2,2)$. 
Type C. The underlying homogeneous space $G / H$ is the matrix group

$$
G=\left(\begin{array}{cccc}
e^{-x_{4}} & 0 & 0 & x_{1} \\
0 & e^{x_{4}} & 0 & x_{2} \\
0 & 0 & 1 & x_{3} \\
0 & 0 & 0 & 1
\end{array}\right)
$$

$(M, g)$ is the space $\mathbb{R}^{4}\left(x_{1}, x_{2}, x_{3}, x_{4}\right)$ with the Lorentzian metric

$$
g=\varepsilon\left(e^{-2 x_{4}} d x_{1}^{2}+e^{2 x_{4}} d x_{2}^{2}\right)+d x_{3} d x_{4} \quad \text { with } \varepsilon= \pm 1 .
$$

The order is $k=3$, and the possible signatures are $(1,3),(3,1)$.

Type D. The underlying homogeneous space is $G / H$, where

$$
G=\left(\begin{array}{ccc}
a & b & x_{1} \\
c & d & x_{2} \\
0 & 0 & 1
\end{array}\right), \quad H=\left(\begin{array}{ccc}
e^{x_{4}} & 0 & 0 \\
0 & e^{-x_{4}} & 0 \\
0 & 0 & 1
\end{array}\right)
$$

with $a d-b c=1 . \quad(M, g)$ is the space $\mathbb{R}^{4}\left(x_{1}, x_{2}, x_{3}, x_{4}\right)$ with the pseudoRiemannian metric

$$
\begin{aligned}
g= & -2 \cosh \left(2 x_{3}\right) \cos \left(2 x_{4}\right) d x_{1} d x_{2}+\lambda\left(d x_{3}^{2}-\cosh ^{2}\left(2 x_{3}\right) d x_{4}^{2}\right) \\
& +\left(\sinh \left(2 x_{3}\right)-\cosh \left(2 x_{3}\right) \sin \left(2 x_{4}\right)\right) d x_{1}^{2} \\
& +\left(\sinh \left(2 x_{3}\right)+\cosh \left(2 x_{3}\right) \sin \left(2 x_{4}\right)\right) d x_{2}^{2},
\end{aligned}
$$

where $\lambda \neq 0$ is a real constant. The order is infinite, and the signature is $(2,2)$.

\section{Curvature of four-dimensional generalized symmetric spa- ce of type $\mathrm{C}$}

Let $(M, g)$ be a four-dimensional generalized symmetric space of type $\mathrm{C}$, and we denote by $\nabla$ and $R$ the Levi-Civita connection and the Riemann curvature tensor of $M$, respectively. Throughout this paper, we will always use the sign convention

$$
R(X, Y)=\nabla_{[X, Y]}-\left[\nabla_{X}, \nabla_{Y}\right]
$$

The Ricci tensor of $(M, g)$ is defined by $\varrho(X, Y)=\operatorname{tr}\{Z \rightarrow R(X, Z) Y\}$.

We shall report the nonvanishing Levi-Civita connection, the Riemann curvature tensor, and the corresponding Ricci tensor with respect to the coordinates vector fields $\left\{\partial_{1}=\frac{\partial}{\partial x_{1}}, \partial_{2}=\frac{\partial}{\partial x_{2}}, \partial_{3}=\frac{\partial}{\partial x_{3}}, \partial_{4}=\frac{\partial}{\partial x_{4}}\right\}$.

Lemma 3.1. Let $M$ be a four-dimensional generalized symmetric space of type $C$. Then the non-vanishing components of the Levi-Civita connection $\nabla$ of $M$ are given by

$$
\begin{array}{ll}
\nabla_{\partial_{1}} \partial_{1}=2 \varepsilon e^{-2 x_{4}} \partial_{3}, & \nabla_{\partial_{1}} \partial_{4}=\nabla_{\partial_{4}} \partial_{1}=-\partial_{1}, \\
\nabla_{\partial_{2}} \partial_{2}=-2 \varepsilon e^{2 x_{4}} \partial_{3}, & \nabla_{\partial_{2}} \partial_{4}=\nabla_{\partial_{4}} \partial_{2}=\partial_{2} .
\end{array}
$$


The only non-zero components of the Riemann curvature tensor $R$ are

$$
\begin{array}{ll}
R_{\partial_{1}, \partial_{4}} \partial_{1}=-2 \varepsilon e^{-2 x_{4}} \partial_{3}, & R_{\partial_{1}, \partial_{4}} \partial_{4}=\partial_{1}, \\
R_{\partial_{2}, \partial_{4}} \partial_{2}=-2 \varepsilon e^{2 x_{4}} \partial_{3}, & R_{\partial_{2}, \partial_{4}} \partial_{4}=\partial_{2},
\end{array}
$$

and the ones obtained by them using the symmetries of the curvature tensor. The non-zero components of the Ricci tensor are given by

$$
\varrho_{\partial_{4}, \partial_{4}}=-2 .
$$

\section{Ricci solitons on four-dimensional generalized symmetric space of type C}

In this section, we analyze the existence of Ricci solitons on the fourdimensional generalized symmetric spaces $(M, g)$ of type C. Let $X=f_{1} \partial_{1}+$ $f_{2} \partial_{2}+f_{3} \partial_{3}+f_{4} \partial_{4}$ be an arbitrary vector field on $(M, g)$, where $f_{1}, \ldots, f_{4}$ are smooth functions of the variables $x_{1}, x_{2}, x_{3}, x_{4}$. The Lie derivative of metric (2.3) with respect to $X$ is given by:

$$
\begin{aligned}
& \left(L_{X} g\right)_{\partial_{1}, \partial_{1}}=2 \varepsilon e^{-2 x_{4}}\left(\partial_{1} f_{1}-f_{4}\right) \\
& \left(L_{X} g\right)_{\partial_{1}, \partial_{2}}=\varepsilon\left(e^{2 x_{4}} \partial_{1} f_{2}+e^{-2 x_{4}} \partial_{2} f_{1}\right), \\
& \left(L_{X} g\right)_{\partial_{1}, \partial_{3}}=\frac{1}{2} \partial_{1} f_{4}+\varepsilon e^{-2 x_{4}} \partial_{3} f_{1}, \\
& \left(L_{X} g\right)_{\partial_{1}, \partial_{4}}=\frac{1}{2} \partial_{1} f_{3}+\varepsilon e^{-2 x_{4}} \partial_{4} f_{1}, \\
& \left(L_{X} g\right)_{\partial_{2}, \partial_{2}}=2 \varepsilon e^{2 x_{4}}\left(f_{4}+\partial_{2} f_{2}\right) \\
& \left(L_{X} g\right)_{\partial_{2}, \partial_{3}}=\frac{1}{2} \partial_{2} f_{4}+\varepsilon e^{2 x_{4}} \partial_{3} f_{2} \\
& \left(L_{X} g\right)_{\partial_{2}, \partial_{4}}=\frac{1}{2} \partial_{2} f_{3}+\varepsilon e^{2 x_{4}} \partial_{4} f_{2}, \\
& \left(L_{X} g\right)_{\partial_{3}, \partial_{3}}=\partial_{3} f_{4} \\
& \left(L_{X} g\right)_{\partial_{3}, \partial_{4}}=\frac{1}{2}\left(\partial_{3} f_{3}+\partial_{4} f_{4}\right) \\
& \left(L_{X} g\right)_{\partial_{4}, \partial_{4}}=\partial_{4} f_{3} .
\end{aligned}
$$

By using (2.3) and (4.1) in (1.1), a standard calculation gives that a fourdimensional generalized symmetric space of type $\mathrm{C}$ is a Ricci soliton if and only if the following system holds:

$$
\begin{aligned}
\partial_{1} f_{1}-f_{4} & =\frac{\lambda}{2}, \\
e^{2 x_{4}} \partial_{1} f_{2}+e^{-2 x_{4}} \partial_{2} f_{1} & =0, \\
\partial_{1} f_{4}+2 \varepsilon e^{-2 x_{4}} \partial_{3} f_{1} & =0, \\
\partial_{1} f_{3}+2 \varepsilon e^{-2 x_{4}} \partial_{4} f_{1} & =0, \\
\partial_{2} f_{2}+f_{4}=\frac{\lambda}{2} &
\end{aligned}
$$




$$
\begin{aligned}
\partial_{2} f_{4}+2 \varepsilon e^{2 x_{4}} \partial_{3} f_{2} & =0 \\
\partial_{2} f_{3}+2 \varepsilon e^{2 x_{4}} \partial_{4} f_{2} & =0 \\
\partial_{3} f_{3}+\partial_{4} f_{4} & =\lambda, \\
\partial_{4} f_{3} & =2, \\
\partial_{3} f_{4} & =0 .
\end{aligned}
$$

Equation (4.11) yields $f_{4}=f_{4}\left(x_{1}, x_{2}, x_{4}\right)$. Deriving equations (4.5) and (4.8) with respect to $x_{4}$, we obtain, since $\partial_{4} f_{3}=2$,

$$
\begin{aligned}
& \partial_{4}^{2} f_{1}=2 \partial_{4} f_{1}, \\
& \partial_{4}^{2} f_{2}=-2 \partial_{4} f_{2} .
\end{aligned}
$$

Hence, integrating, we get

$$
\begin{aligned}
& f_{1}=e^{2 x_{4}} h\left(x_{1}, x_{2}, x_{3}\right)+H\left(x_{1}, x_{2}, x_{3}\right), \\
& f_{2}=e^{-2 x_{4}} k\left(x_{1}, x_{2}, x_{3}\right)+K\left(x_{1}, x_{2}, x_{3}\right),
\end{aligned}
$$

where $h, k, H$, and $K$ are smooth functions depending on $x_{1}, x_{2}, x_{3}$.

Next, from equations (4.2) and (4.6), we have $\partial_{1} f_{1}+\partial_{2} f_{2}=\lambda$. Then we replace $f_{1}$ and $f_{2}$ to obtain

$$
e^{2 x_{4}} \partial_{1} h+e^{-2 x_{4}} \partial_{2} k+\partial_{1} H+\partial_{2} K=\lambda .
$$

Deriving with respect to $x_{4}$, we have

$$
e^{2 x_{4}} \partial_{1} h-e^{-2 x_{4}} \partial_{2} k=0
$$

which, since $x_{4}$ is arbitrary, gives $\partial_{1} h=\partial_{2} k=0$, and so

$$
\partial_{1} H+\partial_{2} K=\lambda \text {. }
$$

We replace $f_{1}$ and $f_{2}$ in equation (4.3) to find

$$
e^{2 x_{4}} \partial_{1} K+e^{-2 x_{4}} \partial_{2} H+\partial_{1} k+\partial_{2} h=0 .
$$

Deriving with respect to $x_{4}$, we find that $\partial_{1} K=\partial_{2} H=0$, which gives

$$
\partial_{1} k+\partial_{2} h=0
$$

Equation in (4.2) yields, since $\partial_{1} h=0, f_{4}=\partial_{1} H-\frac{\lambda}{2}$. Then from equation (4.4), we get

$$
\partial_{1}^{2} H+2 \varepsilon \partial_{3} h+2 \varepsilon e^{-2 x_{4}} \partial_{3} H=0,
$$

which, by derivation with respect to $x_{4}$, gives $\partial_{3} H=0$. Hence, $H$ depends only on $x_{1}$ and thus

$$
\partial_{1}^{2} H+2 \varepsilon \partial_{3} h=0
$$

We derive (4.13) with respect to $x_{1}$, we deduce (since $\partial_{1} K=0$ ) that $\partial_{1}^{2} H=0$. Thus $H=\alpha x_{1}+\beta$ where $\alpha, \beta \in \mathbb{R}$. 
Replacing $H$ in (4.15), we have $\partial_{3} h=0$, and we can conclude that $h$ depends only on $x_{2}$. Because of (4.13), we then have $K=(\lambda-\alpha) x_{2}+\bar{K}\left(x_{3}\right)$, where $\bar{K}$ is a smooth function.

Next, taking the derivative of (4.14) with respect to $x_{2}$, we prove, since $\partial_{2} k=$ 0 , that $h=\gamma x_{2}+\delta$ where $\gamma, \delta \in \mathbb{R}$. Hence, $k=-\gamma x_{1}+\bar{k}\left(x_{3}\right)$, where $\bar{k}$ is a smooth function. Thus, equation (4.6) yields $f_{4}=\alpha-\frac{\lambda}{2}$. Moreover, equation (4.7) implies $\partial_{3} f_{2}=0$, that is, $\bar{k}^{\prime}\left(x_{3}\right) e^{-2 x_{4}}+\bar{K}^{\prime}\left(x_{3}\right)=0$, which gives $\bar{k}^{\prime}\left(x_{3}\right)=\bar{K}^{\prime}\left(x_{3}\right)=0$, and so $\bar{k}\left(x_{3}\right)=a$ and $\bar{K}\left(x_{3}\right)=b$, where $a, b$ are arbitrary real constants. Therefore, (4.12) becomes

$$
\begin{aligned}
& f_{1}=\left(\gamma x_{2}+\delta\right) e^{2 x_{4}}+\alpha x_{1}+\beta, \\
& f_{2}=\left(-\gamma x_{1}+a\right) e^{-2 x_{4}}+(\lambda-\alpha) x_{2}+b .
\end{aligned}
$$

By equations (4.9) and (4.10), we have

$$
f_{3}=\lambda x_{3}+2 x_{4}+\bar{L}\left(x_{1}, x_{2}\right),
$$

where $\bar{L}$ is a smooth function.

By equation (4.8) and using the second equation in (4.16), we prove that $\partial_{2} \bar{L}\left(x_{1}, x_{2}\right)=4 \varepsilon\left(-\gamma x_{1}+a\right)$. Then $\bar{L}\left(x_{1}, x_{2}\right)=4 \varepsilon\left(-\gamma x_{1}+a\right) x_{2}+L\left(x_{1}\right)$, where $L$ is a smooth function, and so $f_{3}=2 x_{4}+\lambda x_{3}+4 \varepsilon\left(-\gamma x_{1}+a\right) x_{2}+L\left(x_{1}\right)$.

Thus, deriving with respect to $x_{1}$ and using equation (4.5) and the first equation in (4.16), we obtain $L\left(x_{1}\right)=-4 \varepsilon \delta x_{1}+\eta$ where $\eta \in \mathbb{R}$. The calculations above proved that the general solution of (4.2)-(4.11) is given by $X=f_{1} \partial_{1}+$ $f_{2} \partial_{2}+f_{3} \partial_{3}+f_{4} \partial_{4}$, where

$$
\begin{aligned}
& f_{1}=\left(\gamma x_{2}+\delta\right) e^{2 x_{4}}+\alpha x_{1}+\beta, \\
& f_{2}=\left(-\gamma x_{1}+a\right) e^{-2 x_{4}}+(\lambda-\alpha) x_{2}+b, \\
& f_{3}=2 x_{4}+\lambda x_{3}+4 \varepsilon\left(-\gamma x_{1}+a\right) x_{2}-4 \varepsilon \delta x_{1}+\eta, \\
& f_{4}=\alpha-\frac{\lambda}{2}
\end{aligned}
$$

for arbitrary real constants $a, b, \alpha, \beta, \gamma, \delta, \eta$. Therefore, the four-dimensional Lorentzian generalized symmetric spaces admit appropriate vector fields for which (1.1) holds. For any value of $\lambda$, we have the following result:

Theorem 4.1. A four-dimensional Lorentzian generalized symmetric space of type $C$ is an expanding, steady and shrinking Ricci soliton.

Now, let $X=\operatorname{grad} h$ be an arbitrary gradient vector field on the fourdimensional Lorentzian generalized symmetric space $(M, g)$ with potential function $h$. Then $X$ is given by

$$
\operatorname{grad} h=\varepsilon e^{2 x_{4}}\left(\partial_{1} h\right) \partial_{1}+\varepsilon e^{-2 x_{4}}\left(\partial_{2} h\right) \partial_{2}+2\left(\partial_{4} h\right) \partial_{3}+2\left(\partial_{3} h\right) \partial_{4} .
$$

By a standard calculation, we prove, using (4.17), that the four-dimensional generalized symmetric space of type $\mathrm{C}$ is a gradient Ricci soliton if and only if

$$
\partial_{1} h=\varepsilon e^{-2 x_{4}}\left[\left(\gamma x_{2}+\delta\right) e^{2 x_{4}}+\alpha x_{1}+\beta\right],
$$




$$
\begin{aligned}
& \partial_{2} h=\varepsilon e^{2 x_{4}}\left[\left(-\gamma x_{1}+a\right) e^{-2 x_{4}}+(\lambda-\alpha) x_{2}+b\right], \\
& \partial_{3} h=\frac{\alpha}{2}-\frac{\lambda}{4}, \\
& \partial_{4} h=x_{4}+\frac{\lambda}{2} x_{3}+2 \varepsilon\left(-\gamma x_{1}+a\right) x_{2}-2 \varepsilon \delta x_{1}+\frac{\eta}{2} .
\end{aligned}
$$

Deriving the last equation in (4.18) with respect to $x_{3}$ and using the third equation in (4.18), we prove that $\lambda=0$. Hence the Ricci soliton is necessarily steady. Next, deriving the first equation in (4.18) with respect to $x_{2}$ and the second equation in (4.18) with respect to $x_{1}$, we get $\gamma=0$. Now, taking the derivative of the last equation in (4.18) with respect to $x_{1}$ and using the derivation of the first equation in (4.18) with respect to $x_{4}$, we obtain $\alpha=\beta=\delta=0$. Then the derivative of the last equation in (4.18) with respect to $x_{2}$ gives ( since $\left.\partial_{2} h=\varepsilon\left(a+b e^{2 x_{4}}\right)\right) a=$ $b=0$. Thus, $h$ depends only on $x_{4}$. Integrating the last equation in (4.18) with respect to $x_{4}$, we deduce that

$$
h=\frac{1}{2}\left(\eta+x_{4}\right) x_{4}+k, \quad k, \eta \in \mathbb{R} .
$$

Thus, we have shown the following corollary.

Corollary 4.2. A four-dimensional Lorentzian generalized symmetric space is a gradient Ricci soliton if and only if it is steady. The potential function $h=$ $h\left(x_{4}\right)$ is given by

$$
h=\frac{1}{2}\left(\eta+x_{4}\right) x_{4}+k, \quad k, \eta \in \mathbb{R} .
$$

Following [6], the existence of solutions to the Ricci soliton equation for different values of $\lambda$ appears to be related to the existence of Ricci and Yamabe solitons on homogeneous spaces.

Thus, by Theorem 4.1, one can deduce that four-dimensional Lorentzian generalized symmetric spaces of type $\mathrm{C}$ are also Yamabe solitons.

\section{References}

[1] P. Baird and L. Danielo, Three-dimensional Ricci solitons which project to surfaces, J. Reine Angew. Math. 608 (2007), 65-91.

[2] W. Batat, M. Brozos-Vazquez, E. García-Río, and S. Gavino-Fernández, Ricci solitons on Lorentzian manifolds with large isometry groups, Bull. Lond. Math. Soc. 43 (2011), 1219-1227.

[3] W. Batat and K. Onda, Four-dimensional pseudo-Riemannian generalized symmetric spaces which are algebraic Ricci solitons, Results Math. 64 (2013), 254-267.

[4] W. Batat and K. Onda, Ricci and Yamabe solitons on second-order symmetric, and plane wave 4-dimensional Lorentzian manifolds, J. Geom. 105 (2014), 561-575.

[5] M. Brozos-Vázquez, G. Calvaruso, E. García-Río, and S. Gavino-Fernández, Threedimensional Lorentzian homogeneous Ricci solitons, Israel J. Math. 188 (2012), $385-403$. 
[6] G. Calvaruso, Oscillator spacetimes are Ricci solitons, Nonlinear Anal. 140 (2016), 254-269.

[7] G. Calvaruso and B. De Leo, Curvature properties of four-dimensional generalized symmetric spaces, J. Geom. 90 (2008), 30-46.

[8] G. Calvaruso and B. De Leo, Ricci solitons on Lorentzian Walker three-manifolds, Acta Math. Hungar. 132 (2011), 269-293.

[9] G. Calvaruso and A. Fino, Four-dimensional pseudo-Riemannian homogeneous Ricci solitons, Int. J. Geom. Methods Mod. Phys. 12 (2015), 1550056, 21 pp.

[10] G. Calvaruso and E. Rosado, Ricci solitons on low-dimensional generalized symmetric spaces, J. Geom. Phys. 112 (2017), 106-117.

[11] L.F. Cerbo, Generic properties of homogeneous Ricci solitons, Adv. Geom. 14 (2014), 225-237.

[12] J. Cerny and O. Kowalski, Classification of generalized symmetric pseudo-Riemannian spaces of dimension $n \leq 4$, Tensor (N.S.) 38 (1982), 256-267.

[13] J. Lauret, Ricci soliton solvmanifolds, J. Reine Angew. Math. 650 (2011), 1-21.

[14] K. Onda, Lorentz Ricci solitons on 3-dimensional Lie groups, Geom. Dedicata 147 (2010), 313-322.

[15] T.L. Payne, The existence of soliton metrics for nilpotent Lie groups, Geom. Dedicata 145 (2010), 71-88.

Received April 5, 2017, revised July 5, 2017.

Amel Bouharis,

Université d'Oran 1 Ahmed Ben Bella, BP 1524, ELM_Naouer 31000, Oran, Algeria,

E-mail: bouharis@yahoo.fr

Bachir Djebbar,

Université des Sciences et de la Technologie d'Oran "Mohamed Boudiaf", BP 1505, Bir El Djir 31000, Oran, Algeria,

E-mail: badj2001@yahoo.fr

\section{Солітони Річчі на лоренцевих чотиривимірних узагальнених симетричних просторах}

Amel Bouharis and Bachir Djebbar

Доведено існування нетривіальних (тобто, неейнштейнівських) солітонів Річчі на чотиривимірних лоренцевих узагальнених симетричних просторах. Більш того, показано, що тільки стійкі солітони Річчі можуть бути градієнтними.

Ключові слова: лоренцева метрика, солітони Річчі, градієнтні солітони Річчі, узагальнені симетричні простори. 